\title{
EL FÚTBOL COMO MEDIACIÓN EDUCATIVA EN EL FORTALECIMIIENTO DE VALORES SOCIALES
}

Soccer as an educational mediation in the strengthening of social values

\author{
Danna Liseth Bedoya Solarte \\ danna.bedoyaooo@endeporte.edu.co \\ Claudia Lorena Serna Olaya \\ claudia.serna@endeporte.edu.co \\ Germán Darío Isaza Gómez \\ german.isaza@endeporte.edu.co \\ Institución Universitaria Escuela Nacional del Deporte \\ Profesional en deporte \\ Colombia
}

\section{RESUMEN}

El deporte se ha posicionado como un protagonista en las comunidades vulnerables, por ello se planteó como objeto el fortalecimiento de valores sociales mediante la práctica del fútbol por medio de las medicaciones educativas en el grupo Brújula de educación flexible de la Institución Educativa Santa Rosa. Metodología: enfoque metodológico mixto, tipo descriptivo con un estudio prospectivo. Se aplicaron diarios en el grupo Brújula, que permitieron conocer las características de los 27 niños y niñas y las situaciones presentadas entre ellos --agresiones físicas y verbales, el irrespeto y la ausencia del manejo de emociones; con los datos obtenidos se generaron estrategias didácticas y pedagógicas para el fortalecimiento de valores sociales (respeto, cooperación), mediante el diseño de un programa deportivo que permite la sana convivencia, el reconocimiento hacia el compañero y, lo más importante, el mantener el respeto y la cooperación entre sí; además se involucraron las capacidades físicas y habilidades motrices propias del fútbol. Conclusión: es necesario impulsar el deporte en las comunidades vulnerables acompañado de valores que les permita mantener relaciones armónicas y una construcción ciudadana en edades tempranas.

PALABRAS CLAVE:

Fortalecimiento de valores, estrategias didácticas y pedagógicas, programa deportivo en fútbol. 


\section{INTRODUCCIÓN}

El artículo aborda el fomento de valores sociales a través del fútbol, con la ayuda de mediaciones educativas que favorecen el proceso de enseñanza-aprendizaje durante la actividad deportiva con los niños y niñas del grupo Brújula de la Institución Educativa Santa Rosa en la Ciudad de Cali. El grupo Brújula posee unas características particulares, entre ellas se destaca la falta de atención, dificultades para el aprendizaje, violencia entre los compañeros, vulnerabilidad social y la desventaja de no poder llevar un proceso de educación convencional.

El objeto del artículo es fortalecer los valores sociales con la práctica del fútbol y las mediaciones educativas en el grupo Brújula de educación flexible de la Institución Educativa Santa Rosa. Para ello se pretende fomentar la vivencia de valores como el respeto y la cooperación, con la ayuda de estrategias inmersas dentro de un programa deportivo en las actividades escolares; del mismo modo, todo esto es favorecido por las mediaciones educativas que se presentan durante la práctica del fútbol y la intervención por parte de los entrenadores, debido a que dichas mediaciones permiten la resolución de conflictos de manera pacífica entre los niños y niñas.

A su vez, participar en una actividad deportiva ya deja de algún modo una enseñanza significativa en los niños. De acuerdo con Blázquez (1995), el deporte educativo: "constituye una verdadera actividad cultural que permite una formación básica, y luego, una formación continua a través del movimiento” (p.27). En este orden de ideas, el deporte se proyecta como un elemento potente para la vivencia de valores y actitudes que redundan en la construcción deportiva, social y humana, el cual proporciona un bienestar colectivo y de calidad.

El diseño metodológico de este trabajo investigativo se determina por presentar un enfoque cualitativo y cuantitativo con un tipo investigación de carácter descriptivo, esto derivado por el tipo de instrumentos utilizados para la recolección de datos, los cuales se obtuvieron a través de entrevistas, diarios de campo, grabaciones y fichas; así mismo, el tipo de estudio elegido es definido por la participación que presentan los investigadores en primera persona durante el desarrollo de las actividades que corresponden al trabajo de grado, orientadas a una población selecta reducida a una muestra de 27 estudiantes pertenecientes al grupo brújula de la Institución Educativa Santa Rosa.

Dentro de los resultados obtenidos se caracterizó el grupo Brújula, conociendo las peculiaridades de la población, además se identificaron las situaciones presentadas dentro del grupo-como las agresiones físicas y verbales, el irrespeto hacia los docentes y compañeros de clase y el rechazo familiar que reciben en sus casas. Por otro lado, se generaron estrategias didácticas y pedagógicas para el fortalecimiento de valores sociales (respeto y cooperación), con el fin de ayudar a mejorar las dificultades identificadas en el grupo y desarrollarlas en conjunto con el programa deportivo. El programa deportivo realizado para los niños brinda ciertas actividades relacionadas con el fútbol, como la estimulación de las habilidades motrices, las capacidades físicas y técnicas apoyadas a través del juego, que a su vez dan como resultado un desarrollo físico y, de modo fundamental, el factor de construcción ciudadana en edades tempranas.

\section{MÉTODO}

Esta investigación tiene como fin fomentar valores sociales en los niños y niñas del grupo Brújula; se realizó de carácter mixto, del que Sampieri (2014) afirma:

Los métodos mixtos representan un conjunto de procesos sistemáticos, empíricos y críticos de investigación e implican la recolección y el análisis de datos cuantitativos y cualitativos, así como su integración y discusión conjunta, para realizar inferencias producto de toda la información recabada (metainferencias) y lograr un mayor entendimiento del fenómeno bajo estudio (p.534). 
El tipo de la investigación fue un estudio descriptivo con tendencia a cohorte prospectivo. La población con la que se realizó el estudio fueron 27 estudiantes del grupo Brújula de la Institución Educativa Santa Rosa, en el Poblado II del distrito de Aguablanca de la ciudad Santiago de Cali, previos consentimientos informados para participar en el estudio, donde se destacan: pertenecer al grupo Brújula, asistir al programa, tener el consentimiento firmado por los padres de familia y, por último, haber llenado las fichas de recolección de datos.

Las técnicas que se utilizaron para recolectar la información para esta investigación fueron entrevistas semiestructuradas para las personas a cargo del grupo, procesos de observación en donde se utilizó los diarios de campo anotando todas las situaciones que ocurrían mientras se estaba realizando las intervenciones con los chicos, fichas de recolección de datos con la información personal de los niños y las anécdotas que hayan ocurrido dentro del salón y, finalmente, la técnica de recolección documental para así llegar a un buena planificación del programa deportivo.

Los métodos utilizados para realizar los análisis pertinentes de la información recolectada fueron el software de Atlas. Ti, ya que ayuda al procesamiento de la información cualitativa, llevando a cabo por medio de este una codificación abierta; la otra técnica utilizada fue el paquete de Office, en donde se realizó la estructura de las estrategias educativas y los medios para cada una de ellas, ya que estas son la base del programa deportivo para fortalecer los valores (respeto y cooperación) con énfasis en fútbol.

\section{RESULTADOS}

Esta investigación tuvo lugar en el distrito de Aguablanca de la ciudad de Cali, en el barrio Poblado II, categorizado como una zona violenta y peligrosa tanto para los habitantes como los visitantes; allí se encuentra la Institución Educativa Santa Rosa y esta ofrece educación primaria y secundaria. El grupo Brújula se caracteriza por ser un grupo con educación flexible, extra-edad, conformado por niños y niñas en condición de vulnerabilidad, producto de las diversas situaciones de conflicto dentro y fuera de la Institución; por ello este trabajo pretende fomentar el desarrollo de habilidades para desarrollar relaciones armónicas entre los estudiantes del grupo Brújula.

En la tabla 1 se presenta la caracterización del grupo Brújula, donde se pueden conocer los porcentajes correspondientes a la edad de los niños y niñas pertenecientes al grupo.

Los porcentajes que aparecen en los resultados muestran que el 33\% de los niños y niñas tienen 11 años; el $22 \%$ tienen 10 y 12 años; el $15 \%$ tienen 9 años y finalmente el $4 \%$ tienen 13 y 15 años.

\begin{tabular}{|c|c|c|c|c|c|}
\hline \multicolumn{7}{|c|}{ CARACTERIZACIÓN DEL GRUPO BRÚJULA } \\
\hline EDADES & NIÑOS & NIÑAS & \multicolumn{2}{|c|}{ COMPOSICIÓN FAMILIAR } \\
\hline 9 & 1 & 3 & TIPO DE FAMILIA & TOTAL & PORCENTAJE \\
\hline 10 & 3 & 3 & Nuclear simple & 7 & $25,93 \%$ \\
\hline 11 & 8 & 1 & Monoparental & 4 & $15 \%$ \\
\hline 12 & 6 & 0 & Monoparental extendida & 8 & $30 \%$ \\
\hline 13 & 1 & 0 & No parental & 5 & $19 \%$ \\
\hline 14 & 0 & 0 & Reconstruida - Binuclear & 1 & $4 \%$ \\
\hline 15 & 1 & 0 & Extensa & 2 & $\mathbf{7 \%}$ \\
\hline TOTAL & $\mathbf{2 0}$ & $\mathbf{7}$ & & $\mathbf{2 7}$ & $\mathbf{1 0 0 , 0 0 \%}$ \\
\hline
\end{tabular}

Tabla 1. Caracterización del Grupo Brújula. Elaboración propia. 
También se expone dentro de la misma tabla la composición familiar de cada uno de los niños del grupo, en donde se logra identificar que tres de cada diez niños integrantes del grupo tienen una familia nuclear simple que está compuesta por papá, mamá e hijos; también se encuentra que tres de diez chicos pertenecen a una familia monoparental extendida que se compone por el padre o madre, hijos y otras personas que tengan parentesco; significa que dos de cada diez niños viven en una familia no parental que se compone por familiares con vínculos de parentesco que realizan función de roles sin la presencia de los padres (tíos, primos, abuelos, hermanos), en donde se puede reflejar que la mayoría de los niños del grupo no tienen a sus dos padres juntos, ya sea que vivan con cualquiera de los dos o que otros familiares cumplan este rol, por lo que puede reflejar una deficiencia en el comportamiento de los niños.

\begin{tabular}{|c|c|c|c|}
\hline \multicolumn{4}{|c|}{ SITUACIONES DE CONFLICTO } \\
\hline SITUACIÓN & TIPO & ESCUELA & CASA \\
\hline \multirow{2}{*}{ AGRESIONES } & FÍSICA & $* * *$ & $*$ \\
\cline { 2 - 4 } & VERBAL & $* * *$ & $*$ \\
\hline \multirow{2}{*}{ IRRESPETO } & HACIA LOS DOCENTES & $* * *$ & \\
\cline { 2 - 4 } & HACIA SUS COMPAÑEROS & $* * *$ & \\
\hline \multirow{2}{*}{ RECHAZO FAMILIAR } & & $*$ & $* *$ \\
\hline
\end{tabular}

Tabla 2. Situaciones de conflicto. Elaboración propia.

Las situaciones de conflicto que se lograron identificar desde la experiencias vividas en el grupo fueron distribuidas en tres categorías, las cuales se obtuvieron de las diferentes técnicas de recolección de datos, arrojando como resultado agresiones físicas y verbales, de lo que Páez, Fernández, Ubillos y Zubieta (2004) afirman: "es una conducta interpersonal cuya intención es herir o causar daño simbólico, verbal o físico a una persona que no desea sufrir esa suerte y que de hecho provoca daño real"(p.533); esto se refleja en algunos relatos:

Durante la realización de las actividades se observa a dos niños que estaban realizando una fila para hacer una de las actividades con el balón de fútbol, uno de los chicos decidió ir y colocarse de primero en la fila, el niño que estaba de primero le dio mucha rabia y empezó a insultar diciendo cosas como "quítate de aquí, pirobo, que yo soy el primero"; como este hizo caso omiso, el niño le siguió diciendo "quítese que aquí voy yo, gonorrea, te voy a meter tu puño si no te quitas". (DC 3:2) ${ }^{1}$

Otra de las situaciones es el irrespeto que se presenta hacia los docentes y compañeros de clases, haciendo que no haya una mejor convivencia entre ellos. Cortázar (2010) afirma:

La práctica del respeto se puede manifestar en el aprecio de las ideas y creencias de los compañeros, en el reconocimiento de las habilidades sin distinción de sexo y edad, igualdad de todos los compañeros sin importar nivel económico, religión y condición física; sin embargo, el respeto abarca desde la propia valoración de la vida y el cuerpo humano, el reconocimiento de la importancia de la tolerancia y el desarrollo moral por el respeto de las cosas ajenas. (p.192)

El respeto es un valor muy importante para la vida, pero en los chicos del grupo se logra reflejar la falta de respeto, hay situaciones en donde se evidencia:

\footnotetext{
${ }^{1} \mathrm{DC}=$ diario de campo 3= número de diario de campo $2=$ número de relato 
En medio de las actividades tres niños del grupo decidieron salir de estas sin pedir permiso previo a los profesores encargados de la actividad, ni tampoco del docente encargado del grupo; el docente, a pesar de los llamados de atención que les estaba haciendo a ellos, lo ignoraron y siguieron caminando hasta llegar a un muro y sentarse ahí saliéndose totalmente de las actividades. (DC 5:3)

Por último, se encuentra una causa y también influye mucho en el comportamiento de los niños y es las relaciones familiares y la convivencia con ellos. Febvre (1961) dice que: "La familia se define como el conjunto de individuos que viven alrededor de un mismo hogar" (p.145), pero en algunos casos los chicos no quieren o no les gusta estar con su familia, ya que viven cosas como lo relata:

Alumno \#5 (2020). Ficha de recolección de datos

Mi hermano sí es diferente, él me pega. En estos días me partió una tabla de la cama. En mi casa todos me pegan, nadie me quiere, además de esto me dicen que me vaya de mi casa, entonces yo salgo de acá para la calle, llegó en la noche a dormir y al otro día es venir a la escuela y de aquí para la calle (F10:1)

Todo esto lleva a que en el colegio no haya una sana convivencia, ya que, en muchas ocasiones, como lo decía el profesor encargado del grupo, ven la escuela como un escape para lo que está sucediendo en sus casas.

Para concluir, todas las situaciones presentadas son las que llevan al conflicto o problemas dentro del salón, lo cual lleva a que no haya una sana convivencia entre ellos, por lo cual se ve afectada en muchas ocasiones la enseñanza y aprendizaje de estos chicos.

Para alcanzar una mejor interacción y comportamiento de los niños dentro de la Institución y el hogar, se emplearon estrategias acordes a cada una de las situaciones conflicto, debido a que permiten la efectividad dentro de la práctica del programa deportivo enfocado en el fútbol.

Estrategia \#1

Estrategia por desarrollar: Inteligencia emocional.

Categoría: Manejo de Emociones.

Medios:

- Expresar lo que sientes al docente encargado en momentos específicos, al igual que a los compañeros.

- Cambio de una actividad a otra.

- Actividades lúdicas con los padres.

- Reforzar las virtudes del niño.

- Trabajar los pensamientos y las actitudes positivas.

\section{Estrategia \#2}

Categoría: Práctica del respeto hacia el otro.

Estrategia por desarrollar: Trabajos grupales e individuales.

${ }^{2} \mathrm{DC}=$ diario de campo $5=$ número de diario de campo $3=$ número de relato

${ }^{3} \mathrm{~F}=$ ficha de recolección de datos $10=$ número de la ficha $1=$ número de relato 
Medios:

- Enseñanza del respeto por medio del cumplimiento de las normas durante las actividades.

- Trabajo en equipo (juegos cooperativos).

\section{Aptitudes humanas necesarias para el perfil de un entrenador.}

Las estrategias estipuladas anteriormente tienen relación directa con las habilidades y/o cualidades que tiene el entrenador o educador para proceder a un proceso de enseñanza óptimo y un manejo de las categorías tratadas que permiten su cumplimiento.

Dentro de los procesos de enseñanza en el medio deportivo no se puede afirmar cuáles serían las habilidades o cualidades precisas que un entrenador o educador debe tener, puesto que cada persona tiene habilidades y cualidades propias que lo diferencian y lo caracterizan dentro de un entorno social; del mismo modo, no significa que no puedan existir ciertos factores que permitan potenciar los procesos de enseñanza y más cuando se hace referencia al deporte como medio clave para fomentar valores sociales en niños y niñas que aún se encuentran en su etapa escolar y tienen como característica principal el pertenecer a un ambiente en situación de vulnerabilidad. En los entornos vulnerables siempre será importante el uso de las mediaciones educativas como medio para la resolución de conflictos, dado a que ayudan a crear un entorno más pacífico donde no se naturalizan las situaciones de conflicto, sino que se da paso a una solución rápida y eficaz que permita a los niños y niñas entender el valor del respeto, el perdón y la aceptación de los errores que solo serán posibles si el entrenador hace uso de cierta mediación.

Por ello, para la práctica de las estrategias en el programa deportivo es necesario que un entrenador tenga ciertas habilidades como:

- Manejo de grupo.

- Actitud positiva.

- Buen manejo de emociones y sentimientos.

- Sentido humano.

- Resolución de conflictos.

- Pensamiento crítico.

- Comunicación asertiva en los diferentes entornos.

\section{DISCUSIÓN Y CONCLUSIÓN}

Esta investigación tiene como propósito principal el diseño de un programa deportivo para el fortalecimiento de valores sociales (respeto y cooperación), con el fin de favorecer a los niños del grupo Brújula, teniendo presente que hacen parte de una comunidad en situación de vulnerabilidad.

Para comprender si existía una necesidad respecto al fortalecimiento de valores se hacía fundamental el conocimiento de las situaciones de conflicto en el grupo, donde se comprobó que los niños y niñas pertenecientes a Brújula manifestaban una gran desventaja frente a la práctica de valores, deteriorando a su vez la convivencia entre ellos. Isaza, Rengifo y Cano (2018) afirman:

El educador o entrenador deportivo debe observar, identificar y crear estrategias a partir de la realidad que viven los niños, niñas y jóvenes; donde ellos se sientan involucrados en su propio proceso de 
aprendizaje, es necesario conocer sus dificultades, sus necesidades, para poder intervenir adecuadamente los procesos de formación a partir de la vivencia de los valores. (p.5)

La cita anterior nos confirma que para abordar una población es importante conocer las ventajas y desventajas que esta posee, dado a que facilitan la comprensión de las necesidades a tratar (Gómez, 2016). El conocimiento del grupo Brújula permitió la creación del programa deportivo de esta investigación, que a través de las estrategias propuestas permiten que a futuro este sea aplicado por medio de las mediaciones educativas, dado a que reducen el conflicto para favorecer las relaciones interpersonales de los niños y niñas. Fraile (2004) ratifica:

Si queremos que el deporte en edad escolar ayude a desarrollar no únicamente la constancia, el esfuerzo, la superación y la disciplina, sino el trabajo en equipo, la tolerancia, la cooperación, la solidaridad, la igualdad, la autoestima, la responsabilidad y el respeto, entre otros muchos valores, deberemos apostar por una legislación que asegure la profesionalidad de los técnicos y el contenido educativo de los programas deportivos. (p.198)

En el mismo sentido, el programa deportivo se pretende realizar con estrategias inmersas en las actividades del fútbol para proporcionar los valores de respeto y cooperación, teniendo como pilar las normas del juego y el trabajo en equipo, y así al momento de su aplicación poder fortalecer progresivamente ciertos valores que son esenciales para el desarrollo social y personal de cada uno de los niños y niñas que se encuentran en situación de vulnerabilidad; el deporte es una oportunidad para aprender producir cambios en cuanto al entorno social, siempre y cuando exista una pedagogía óptima para el proceso de enseñanza y aprendizaje en las edades tempranas.

Finalmente, el programa aspira contar con ciertas características que benefician al grupo Brújula, tales como la interacción sana, la vivencia deportiva, el reconocimiento hacia el compañero, aumento en el rendimiento físico y por último el fortalecimiento de los valores sociales. Es importante reconocer que estos niños hacen parte de una educación flexible, que les posibilita continuar sus estudios en medio de sus particularidades individuales, siendo el fútbol la mejor herramienta para motivarlos en el proceso educativo, teniendo en cuenta las necesidades de los niños y niñas que impulsan a que el programa deportivo sea mejor para ellos.

Para concluir, se plantea procesos de transformación social a través del deporte, y con ayuda de mediaciones educativas es necesario conocer la realidad que viven día a día los niños y niñas en la Institución Educativa Santa Rosa y en sus alrededores para así poder dar paso al diseño del programa deportivo con fortalecimiento en valores (respeto y cooperación) mediante la práctica del fútbol. El programa se fundamenta de acuerdo con las características y el reconocimiento de las situaciones que actualmente presenta el grupo Brújula y que afecta en gran medida la sana convivencia y el desarrollo de estos, por ende, se entiende la necesidad de atención y apoyo del cual carecen dentro de su etapa escolar.

El conocer los conflictos que enfrentan a diario los niños y niñas de Brújula permite llegar a una amplia perspectiva para el aporte de la construcción de estrategias educativas que contribuyen al planteamiento y desarrollo de cada una de las mediaciones y de los medios propuestos para alcanzar los objetivos planteados, logrando así cada uno de los parámetros propuestos dentro de este trabajo de grado.

El programa deportivo es relevante para los profesionales en deporte e investigadores, dado a que promueve la práctica de valores y la ventaja de disolver los conflictos a través de las mediaciones educativas dentro de las actividades del fútbol. Significa un aporte al mejoramiento individual y colectivo para los niños que se encuentren en comunidades vulnerables y se encuentran en la etapa de desarrollo físico y personal. 
Impulsar la práctica del deporte como medio educativo es una buena forma de contribuir en la sociedad y generar cambios en los niños y niñas para que puedan manejar de la mejor forma las situaciones que se les presente, además de promover el pensamiento reflexivo de lo que hacen en su diario vivir, teniendo presente si aquello es productivo o no para la vida de ellos mismos; el deporte es una ventaja para la sociedad porque da paso a la vida saludable a través de la creación de nuevas amistades, el aprovechamiento del tiempo libre, el aprendizaje de nuevas habilidades, y el uso de la creatividad que influyen en la vida de cada persona dando la oportunidad de disfrutar y aprender del mismo.

\section{REFERENCIAS BIBLIOGRÁFICAS}

Blázquez, D., Amador, F., \& Batalla, A. (1995). La iniciación deportiva y el deporte escolar. Barcelona: Inde. Cortázar, J. (2010). El respeto: ¿Un valor en crisis? Revista 714 blogspot.

Febvre, L. (1961). La tierra y la evolución humana. Introducción geográfica a la historia. Colección la evolución de la humanidad. México: Editorial Hispanoamericana.

Fraile, A. (2004). El deporte escolar en el siglo XXI. Análisis y debate desde una perspectiva europea. Barcelona: Graó. Gómez, G. (2016). Proyecto: Misión ENDeporte. RedPensar, 5(2), 1-16.

Isaza, G., Rengifo, R., \& Cano, J. (2018). Experiencias pedagógicas significativas de transformación social y construcción de paz a través de la formación deportiva en la ciudad de Santiago de Cali, Colombia. Revista Internacional de Educación y Aprendizaje, 201-207.

Páez, D., Fernández, I., Ubillos, S., \& Zubieta, E. (2004). Psicología social, cultura y educación. Pearson Prentice Hall.

Sampieri, R. (2014). Metodología de la investigación. México: Mcgraw-Hill / Interamericana Editores, S.A. de C.V. 